\title{
Modeling of the elastic characteristics of a long- fiber reinforced composite with an arbitrary orientation of the reinforcing fibers
}

\author{
Victor P. Pavlov ${ }^{1}$, Vilina M. Kudoyarova ${ }^{1, *}$, and Alexander A. Philippov ${ }^{1}$ \\ ${ }^{1}$ Ufa State Aviation Technical University, st. K. Marcsa,12, Ufa, 450000, Russian Federation
}

\begin{abstract}
Currently, the question of the best scheme for constructing a mathematical model of a homogeneous anisotropic elastic material equivalent to fiber reinforced plastic composite (FRP) with arbitrary laminate stacking sequence configuration, have been remained open. A new method for the theoretical prediction of anisotropic elastic characteristics of material equivalent to a given FRP is suggested in this paper. Results are obtained for representative volume element (RV) which has been cut out in three different ways from FRP. Calculations for a specific FRP have shown that the FRP replacement by a homogeneous anisotropic material equivalent to it leads to an error order $10 \%$ for the elastic properties.
\end{abstract}

\section{Introduction}

The problem of FRP strength and rigidity calculation is especially important in the design of modern constructions widely used in various engineering fields. It is necessary to know the elastic properties of the applied FRP to solve such a problem. The experimental method for determining FRP properties is the most reliable [1-7], but it is very laborious and does not allow determining the mechanical characteristics of all applicable FRP reinforcement schemes. Therefore, simulation of the FTP mechanical characteristics for various structures [8-19] and determination of the FRP effective characteristics of elasticity, which allow the effective methods of the theory of elasticity for anisotropic body to be used to evaluate the stiffness and strength of FRP structures, is more rational [20] in many cases.

Currently, the question of the best scheme for constructing a mathematical model of a homogeneous anisotropic elastic material equivalent to FRP with arbitrary laminate stacking sequence configuration, have been remained open. One solution is proposed in this paper.

\section{Problem definition}

The paper considers FRP composite, which may have any FRP reinforcement scheme and any elasticity characteristics of its reinforcement and matrix components.

*Corresponding author: Kudoyarova@inbox.ru 
The task to assess the feasibility of the proposed method intended to determine the elasticity characteristics of a homogeneous anisotropic material equivalent to FRP composite under consideration with a given structure, is set in the paper. The method is based on the equality of elastic strain energies in real FRP composite and equivalent anisotropic material with identical deformations of their representative elements.

\section{FRP composite for determining the effective elastic characteristics}

Let us consider a unidirectional FRP composite having straight reinforcing fibers with a diameter $d=10 \mathrm{mic}$. The distance between the fibers is equal to $c=5$ mic. The FRP reinforcement ratio of the composite equals to $\Psi=0,348$. Modelling of FRP properties is performed on its representative volumes (RV) are cut out in various ways.

The RV has the shape of a cube with the following sizes: $a_{x}=a_{y}=a_{z}=15$ mic.

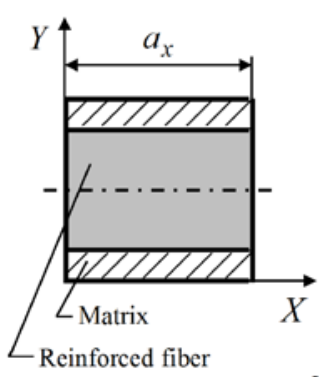

a)

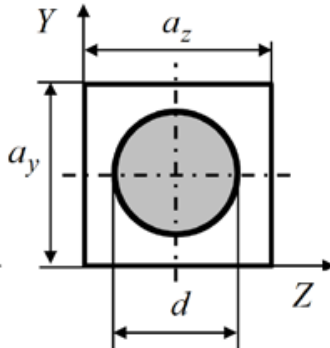

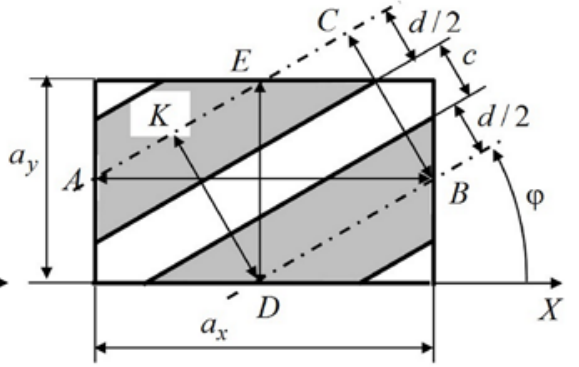

b)

Fig. 1. a) RV for a FRP; b) RV of the same FRP cut out when reinforcing fiber makes an angle $\varphi$ with the $X$-axis

With accordance to fig. 1 , (b) $a_{x}=(d+c) / \sin \varphi, a_{y}=(d+c) / \cos \varphi, a_{z}=15$ mic.

\section{The condition for the equivalence of elastic characteristics for FRP and an equivalent homogeneous material}

The equivalent material model (EM) for FRP is adopted in the form of Hooke's law for a homogeneous anisotropic body:

$$
\left\{\begin{array}{l}
\sigma_{x}=E_{11} \varepsilon_{x}+E_{12} \varepsilon_{y}+E_{13} \varepsilon_{z}+E_{14} \gamma_{x y}+E_{15} \gamma_{y z}+E_{16} \gamma_{x z}, \\
\sigma_{y}=E_{21} \varepsilon_{x}+E_{22} \varepsilon_{y}+E_{23} \varepsilon_{z}+E_{24} \gamma_{x y}+E_{25} \gamma_{y z}+E_{26} \gamma_{x z}, \\
\sigma_{z}=E_{31} \varepsilon_{x}+E_{32} \varepsilon_{y}+E_{33} \varepsilon_{z}+E_{34} \gamma_{x y}+E_{35} \gamma_{y z}+E_{36} \gamma_{x z}, \\
\tau_{x y}=E_{41} \varepsilon_{x}+E_{42} \varepsilon_{y}+E_{43} \varepsilon_{z}+E_{44} \gamma_{x y}+E_{45} \gamma_{y z}+E_{46} \gamma_{x z}, \\
\tau_{y z}=E_{51} \varepsilon_{x}+E_{52} \varepsilon_{y}+E_{53} \varepsilon_{z}+E_{54} \gamma_{x y}+E_{55} \gamma_{y z}+E_{56} \gamma_{x z}, \\
\tau_{x z}=E_{61} \varepsilon_{x}+E_{62} \varepsilon_{y}+E_{63} \varepsilon_{z}+E_{64} \gamma_{x y}+E_{65} \gamma_{y z}+E_{66} \gamma_{x z},
\end{array}\right.
$$

Representative elements for FRP composite and for EM are being considered as a rectangular parallelepiped shape with $a_{x} \times a_{y} \times a_{z}$ dimensions (fig. 1) and then we are assuming that their deformation is similar of their external surfaces deformation.

In accordance with it is assumed that FRP and RV have equivalent elasticity characteristics under condition for identical deformations the specific potential energies of elastic deformations accumulated in FRP and RV are the same. 
The implementation of this approach is being effected in two phases.

\subsection{First phase}

Six numerical experiments were being performed at this phase. In the first experiment, RV with dimensions $a_{x} \times a_{y} \times a_{z}$ is deformed $\varepsilon_{x} \neq 0$ under zero other deformations. The displacement vectors $\bar{\delta}_{m}, m=1,2 \ldots, M$ and the external force vectors $\bar{F}_{m}, m=1,2 \ldots, M$ for all $M$ nodes of the RV have been determined using ANSYS based on the finite element method (FEM). The work of all external forces applied to the RV is determined on their basis. The work determines the internal energy of the elastic deformation in the RV under consideration:

$$
A\left(\varepsilon_{x}\right)=\frac{1}{2} \sum_{m=1}^{M} \bar{F}_{m} \cdot \bar{\delta}_{m} .
$$

Further, the PV is subjected to the same deformation for EM, having the same dimensions, and the potential energy accumulated in it is determined with formula [20]:

$$
U\left(\varepsilon_{x}\right)=\frac{1}{2} \varepsilon_{x} \sigma_{x} V=\frac{1}{2} E_{11} \varepsilon_{x}^{2} V
$$

where $V=a_{x} a_{y} a_{z}$ - the volume of the RV.

Equating $A\left(\varepsilon_{x}\right)$ and $U\left(\varepsilon_{x}\right)$, we obtain a formula for computing $E_{11}$ :

$$
E_{11}=\frac{1}{\varepsilon_{x}^{2}} \frac{1}{V} \sum_{m=1}^{M} \bar{F}_{m} \cdot \bar{\delta}_{m} .
$$

In the same way, the coefficients of elasticity $E_{k k}, k=2, \ldots, 6$ are being determined by setting zero values for deformations $\varepsilon_{y}, \varepsilon_{z}, \gamma_{x y}, \gamma_{y z}, \gamma_{x z}$.

\subsection{Second phase}

External loads are being calculated with synchronous action of two deformations to determine the elastic characteristics $E_{k n}$ at $k \neq n$. For example, $\varepsilon_{x} \neq 0, \varepsilon_{y} \neq 0$ are being set, and the remaining deformations $\varepsilon_{z}=\gamma_{x y}=\gamma_{y z}=\gamma_{x z}=0$. In this case, on the basis of (1), we write

$$
\sigma_{x}=E_{11} \varepsilon_{x}+E_{12} \varepsilon_{y}, \sigma_{y}=E_{21} \varepsilon_{x}+E_{22} \varepsilon_{y},
$$

and for EM model RV the potential energy of elastic deformation is being defined:

$$
U\left(\varepsilon_{x}, \varepsilon_{y}\right)=\frac{1}{2}\left(\varepsilon_{x} \sigma_{x}+\varepsilon_{y} \sigma_{y}\right) V=\frac{1}{2}\left(E_{11} \varepsilon_{x}^{2}+2 E_{12} \varepsilon_{x} \varepsilon_{y}+E_{22} \varepsilon_{y}^{2}\right) V
$$

Equating (6) with the energy obtained from the FEM analysis, we are writing:

$$
\frac{1}{2}\left(E_{11} \varepsilon_{x}^{2}+2 E_{12} \varepsilon_{x} \varepsilon_{y}+E_{22} \varepsilon_{y}^{2}\right) V=\frac{1}{2} \sum_{m=1}^{M} \bar{F}_{m} \cdot \bar{\delta}_{m}
$$

Finally, we are getting 


$$
E_{12}=\frac{1}{2} \frac{1}{\varepsilon_{x} \varepsilon_{y}}\left(\frac{1}{V} \sum_{m=1}^{M} \bar{\delta}_{m} \cdot \bar{F}_{m}-E_{11} \varepsilon_{x}^{2}-E_{22} \varepsilon_{y}^{2}\right) .
$$

The values of $E_{11}$ and $E_{22}$ are calculated in the experiments in phase 1 . Therefore, the quantity $E_{12}$ is uniquely determined by formula (8).

Proceeding in a similar way, all the quantities $E_{k n}$ at $k \neq n$ will be calculated.

\section{FEM results}

According to the method described above, the elastic characteristics of the FRP under consideration have been found out for three cuttings of RV, determined in accordance with fig. 1, (b) for angles $\varphi=0^{\circ}, 30^{\circ}, 45^{\circ}$. Numerical calculations have been performed using ANSYS with 30 thousand elements. The results are presented in the form of $\boldsymbol{E}_{\varphi}$ matrices of FRP stiffness coefficients having a symmetric structure.

$$
\begin{aligned}
& \boldsymbol{E}_{0}=\left(\begin{array}{cccccc}
8.19 e+10 & 7.48 e+09 & 7.48 e+09 & 0 & 0 & 0 \\
& 2.29 e+10 & 5.38 e+09 & 0 & 0 & 0 \\
& & 2.29 e+10 & 0 & 0 & 0 \\
& & & 1.56 e+10 & 0 & 0 \\
& & & & 8.57 e+09 & 0 \\
& & & & & 1.56 e+10
\end{array}\right) \\
& \boldsymbol{E}_{30}=\left(\begin{array}{cccccc}
5.89 e+10 & 1.18 e+10 & 7.51 e+09 & 1.79 e+10 & 1.02 e+06 & -2.13 e+05 \\
& 2.85 e+10 & 5.42 e+10 & 1.04 e+10 & 8.69 e+05 & -4.29 e+05 \\
& & 2.66 e+10 & 1.43 e+09 & 1.84 e+06 & 4.69 e+04 \\
& & & 1.95 e+10 & -1.14 e+05 & 3.40 e+05 \\
& & & & 9.60 e+09 & 3.69 e+09 \\
& & & & & 1.39 e+10
\end{array}\right) \\
& \boldsymbol{E}_{45}=\left(\begin{array}{cccccc}
4.29 e+10 & 1.32 e+10 & 6.63 e+09 & 1.59 e+10 & -2.62 e+05 & 5.59 e+04 \\
& 4.29 e+10 & 6.63 e+09 & 1.59 e+10 & -5.60 e+04 & 2.72 e+04 \\
& & 2.71 e+10 & 1.77 e+09 & -6.14 e+04 & 6.36 e+04 \\
& & & 2.19 e+10 & 1.20 e+04 & -1.20 e+04 \\
& & & & 1.24 e+10 & 4.44 e+09 \\
& & & & & 1.24 e+10
\end{array}\right)
\end{aligned}
$$

\section{Results comparison}

This paper compares the FRP stiffness characteristics calculated on the FEM basis obtained at different angles of the RV cutting from the FRP body with the stiffness characteristics of an equivalent anisotropic material calculated on the basis of the theory of elasticity. At calculations based on theory of elasticity the stiffness characteristics obtained on the basis of the FEM for a representative element having a reinforcing fiber directed along the $X$-axis (fig. 1) are taken as initial characteristics 
The defining relations of the theory of elasticity for an anisotropic body in an orthogonal coordinate system are being written in the form [20]:

$$
\sigma_{i j}=C_{i j k l} \varepsilon_{k}, i, j, k, l=1,2,3,
$$

where $C_{i j k l}$ - are the tensor components of elastic constants $\left(C_{i j k l}\right)$, which have an independent component in the most general case of anisotropy for a linearly elastic body.

The components $C_{i j k l}^{\prime}$ of a given tensor in a coordinate system $x_{\alpha}^{\prime}, \alpha=1,2,3$ has the same origin as the system $x_{i}, i=1,2,3$, but rotated relatively are by [20]:

$$
C_{i j k l}^{\prime}=n_{i \alpha} n_{j \beta} n_{k \gamma} n_{l \delta} C_{\alpha \beta \gamma \delta},
$$

where $n_{i \alpha}, \ldots-$ are the cosines of the angles between the axes $x_{i}^{\prime}$ and the axes $x_{\alpha}$.

On the basis of (13) the matrices $\tilde{\boldsymbol{E}}_{30}, \tilde{\boldsymbol{E}}_{45}$ of RV stiffness coefficients had been determined at the angles $\varphi=30^{\circ}, 45^{\circ}$, and then matrices $\boldsymbol{\Delta}_{30}, \boldsymbol{\Delta}_{45}$ of relative discrepancies were calculated by formula

$$
\boldsymbol{\Delta}_{\varphi}=\frac{\boldsymbol{E}_{\varphi}-\tilde{\boldsymbol{E}}_{\varphi}}{E_{\max }^{\varphi}}, \quad \varphi=30^{\circ}, 45^{\circ},
$$

where $E_{\max }^{\varphi}-$ is the maximum modulus component of the matrix $\boldsymbol{E}_{\varphi}$.

Finally we have got:

$$
\begin{aligned}
& \Delta_{30}=\left(\begin{array}{cccccc}
-5.38 \mathrm{e}-02 & -1.44 \mathrm{e}-02 & 9.19 \mathrm{e}-03 & 3.72 \mathrm{e}-02 & 1.91 \mathrm{e}-06 & -2.62 \mathrm{e}-06 \\
& -6.90 \mathrm{e}-02 & -8.32 \mathrm{e}-03 & 1.09 \mathrm{e}-02 & -1.34 \mathrm{e}-06 & -8.90 \mathrm{e}-07 \\
& & 6.15 \mathrm{e}-02 & 8.75 \mathrm{e}-03 & -6.44 \mathrm{e}-06 & -6.61 \mathrm{e}-06 \\
& & & -2.24 \mathrm{e}-02 & -1.54 \mathrm{e}-06 & -7.07 \mathrm{e}-07 \\
& & & & -1.27 \mathrm{e}-02 & 1.08 \mathrm{e}-02 \\
& & & & & 7.42 \mathrm{e}-04
\end{array}\right) \\
& \Delta_{45}=\left(\begin{array}{cccccc}
-6.34 \mathrm{e}-02 & -2.58 \mathrm{e}-02 & 4.63 \mathrm{e}-03 & 2.66 \mathrm{e}-02 & 2.19 \mathrm{e}-06 & -3.54 \mathrm{e}-06 \\
& -6.34 \mathrm{e}-02 & 4.63 \mathrm{e}-03 & 2.66 \mathrm{e}-02 & 3.54 \mathrm{e}-06 & -2.17 \mathrm{e}-06 \\
& & 9.90 \mathrm{e}-02 & 1.69 \mathrm{e}-02 & 6.52 \mathrm{e}-06 & -6.47 \mathrm{e}-06 \\
& & & -1.38 \mathrm{e}-02 & -4.37 \mathrm{e}-07 & 4.39 \mathrm{e}-07 \\
& & & & 6.96 \mathrm{e}-03 & 2.12 \mathrm{e}-02 \\
& & & & & 6.96 \mathrm{e}-03
\end{array}\right)
\end{aligned}
$$

From (15) and (16) we see that the maximum relative divergence for the rigidity characteristics for $\varphi=30^{\circ}$ is equal to $6,9 \cdot 10^{-2} \cdot 100 \%=6,9 \%$, and for $\varphi=45^{\circ}$ is equal to $9,9 \cdot 10^{-2} \cdot 100 \%=9,9 \%$.

\section{Conclusion}

- A criterion of equivalence with respect to elasticity characteristics between FRP composite of arbitrary structure and a homogeneous anisotropic material based on the 
equality of internal elastic-strain energies at the same RV deformation and EM has been proposed,

- The paper has suggested the RV structure of FRP composite on a phthalonitrile binder reinforced with carbon fibers. This structure is convenient for testing the determination method of the effective characteristics for FRP,

- A methodology for calculating the stiffness coefficients of a homogeneous anisotropic material equivalent to a FRP composite having a phthalonitrile matrix reinforced with carbon fibers has been developed and tested by FEM using ANSYS. Results have been obtained for RV which had been cut out in three different ways from FRP composite,

- A new method for quantifying the accuracy of the correspondence between the FRP effective stiffness characteristics calculated by FEM and the characteristics obtained within the framework of the theory of elasticity for an anisotropic body is suggested,

- Calculations for a specific FRP composite have shown that the FRP replacement by a homogeneous anisotropic material equivalent to it leads to an error order $10 \%$ for the elastic properties.

The authors acknowledge receiving support base part of funded research program of Russian Foundation for Basic Research (RFBR) and Government of the Republic of Bashkortostan in the framework of a scientific project №_17-48-020824_p_a.

\section{References}

1. V. P. Pavlov, Creep of polymer composite materials at variable elevated temperatures. Experimental research and mathematical modeling, (UGATU, 2004) (in Russ.)

2. V. P. Pavlov, Thermal deformation, strength and thermal viscoelasticity of GRP at a high temperature-variable temperature under thermal destruction conditions. Experimental research and mathematical modeling, (UGATU, 2004) (in Russ.)

3. E. K. Ashkenazi, E. V. Ganov, Anisotropy of structural materials: Handbook. (Mashinostroenie, 1972) (in Russ.)

4. Composite materials, vol. 2, Mechanics of composite materials, (Mir, 1978) (in Russ.)

5. Composite materials, vol. 7, Analysis and design of structures, (Mashinostroenie, 1978)

6. Yu. M. Tarnopol'skij, I. G. Zhigun, V. A. Polyakov, Spatially-reinforced composite materials: Handbook, (Mashinostroenie, 1987) (in Russ.)

7. Yu. V. Sokolkin, A. A. Tashkinov, Mechanics of deformation and destruction of structurally heterogeneous bodies, (Nauka, 1984) (in Russ.)

8. V. P. Pavlov, E. M. Nusratullin, A. A. Philippov, Vestnik UGATU 14, 4 (2010)

9. V. P. Pavlov, E. M. Nusratullin, A. A. Philippov, Vestnik UGATU 15, 4 (2011)

10. V. V. Vasil'ev, Mechanics of composite structures, (Mashinostroenie, 1988) (in Russ.)

11. V. P. Pavlov, E. M. Nusratullin, A. A. Philippov, I. Z. Mukhamedova, Izvestia KGASU 3 (2012) (in Russ.)

12. V. P. Pavlov, E. M. Nusratullin, A. A. Philippov, I. Z. Mukhamedova, Vestnik UGATU 20, 3 (2016) (in Russ.)

13. Victor P. Pavlov, Vilina M. Kudoyarova and Alexander A. Philippov, MATEC Web Conf. 129, (2017)

14. S. A. Lur'e, Yusefi Shakhram, Mekh. kompozic. mate i konstr. 3, 4 (1996) (in Russ.)

15. Yu. I. Dimitrienko, A. P. Sokolov, Vestnik MGTU 2, (2008) (in Russ.) 
16. Yu. I. Dimitrienko, A. P. Sokolov, Vestnik MGTU 2, (2008) (in Russ.)

17. Yu. I. Dimitrienko, A. P. Sokolov, Informacionnye tekhnologii 8, (2008) (in Russ.)

18. Yu. I. Dimitrienko, A. P. Sokolov, Informacionnye tekhnologii 8, (2008) (in Russ.)

19. Yu. I. Dimitrienko, A. P. Sokolov, A. I. Kashkarov, Vestnik MGTU (2004) (in Russ.)

20. S. G. Lekhnickij, Theory of elasticity of an anisotropic body, (Nauka, 1977) (in Russ.) 